\title{
Associations of peak shifts in age-prevalence for human malarias with bednet coverage
}

\author{
T. Smith ${ }^{1,2}$, J. L. K. Hii ${ }^{1,3}$, B. Genton ${ }^{1,2}$, I. Müller ${ }^{2}$, M. Booth ${ }^{2}$, N. Gibson ${ }^{1 \dagger}$, A. Narara ${ }^{1}$ and \\ M. P. Alpers ${ }^{1}{ }^{i}$ Papua New Guinea Institute of Medical Research, P.O. Box 378, Madang, Papua New Guinea; ${ }^{2}$ Swiss \\ Tropical Institute, Socinstrasse 57, Postfach CH-4002, Basel, Switzerland; ${ }^{3}$ School of Public Health and Tropical Medicine, \\ fames Cook University, Townswille, Queenstand 4811, Australia
}

\begin{abstract}
Effects of bednet coverage (C) on prevalence of malaria were analysed using data from 1990-92 from 9 Papua New Guinean villages. Effects of coverage varied by age, resulting in a shift in age of peak prevalence from $4.7(\mathrm{C}=0 \%)$ to $11.6(\mathrm{C}=100 \%)$ years for Plasmodium falciparum, from 3.4 to 4.9 years for $P$. vivax and from 11.0 to 16.8 years for $P$. malariae. In small areas with no bednets the age distribution of $P$. falciparum parasitaemia was like that of a holoendemic area. Where coverage was complete the pattern corresponded to mesoendemicity. Thus, protracted use of bednets can result in profound changes in the endemicity of malaria even when coverage is incomplete and without insecticide treatment. Average entomological inoculation rates (EIRs) estimated from indoor landing rates on individuals without bednets were 35,12 and 10 infectious bites per person per annum for $P$. falciparum, $P$. vivax and $P$. malariae, respectively. Logistic regression analyses indicated that the EIR estimate for $P$. falcipartim was related to prevalence of this species independently of effects of bednet coverage. However, the recent EIR still accounted for much less variation than did the bednets. A similar pattern was seen for $P$. malariae, while there were no significant relationships between the recent EIR and the parasite positivity for $P$. vivax. It is concluded that short-term variations in inoculation rate are not important determinants of parasite prevalence in this population.
\end{abstract}

Keywords: malaria, Plasmodium falciparum, Plasmodium vivax, Plasmodizm malariae, prevalence, age, endemicity, bednets, entomological inoculation rates, Papua New Guinea

\section{Introduction}

Bednets have long been used as protection against mosquitoes (LINDSAY \& GiBSON, 1988), but have only recently become a cornerstone of malaria control strategies. This renewed interest is because insecticide-treated nets (ITNs) have been shown to substantially reduce malaria thorbidity atd mortality (reviewed by LENGELER, 1998). A major operational concern for ITN programmes is that high rates of re-treatment are difficult to achieve. Hence it is important to understand how well the nets will function once the insecticide has been lost. Observational studies (e.g., CAMPBEI.L et al., 1987) have suggested that untreated nets (UTNs) also protect against malaria; however, the only randomized controlled trial (RCT) found no significant effects (SNOW et al., 1988).

In contrast to the results of that RCT, we previously observed that the prevalence of Plasmodium falciparum parasitaemia was strongly negatively correlated with levels of UTN use measured at the village level in the Wosera area of Papua New Guinea (GENTON et al, 1994, 1995). There was a stronger relationship between infection prevalence and the proportion using UTNs of persons living within $25 \mathrm{~m}$ (C), than there was between infection status and self-reported bednet use at the individual level (HII et al., 2001). This is probably because the bednets act by reducing the vectorial capacity of the mosquitoes, especially their survival rate, rather than because of individual protection (HII et al., 2001).

The UTNs in the Wosera had been present for several years at the time of the study, so a possible explanation for differences between our results and those from The Gambia (SNOw et al., 1988) is that effects of bednets accumulate only slowly. Long-term reduction in the transmission rate of a parasite can have cumulative effects, characteristically increasing the age at which the maximum prevalence is reached (ANDERSON \& MAY, 1992) and reducing the actual peak prevalence. Together these variables show the strength of the peak

\section{IDeceased.}

Address for correspondence: Dr T. Smith, Department of Public Health and Epidemiology, Swiss Tropical Institute, Socinstrasse 57, Postfach CH-4002, Basel, Switzerland; e-mail smith@ubaclu.unibas.ch shift in prevalence (Wooluouse, 1998), and thus indicate the effect of bednet coverage on exposure to parasites and hence acquired immunity.

We have now analysed age-variation in patterns of malaria infection in the Wosera, for villages with different levels of UTN coverage, using a statistical approach based on the malaria model of ARON (1983). This analysis enabled us to estimate the strength of the peak shift associated with UTN use for each malaria species. Using a geographical information system and spatial statistical models, we also estimated human biting rates, $m a$, for the 6 different vector species in each of 205 small groups of houses, for each of 6 perjods of 4 months. We multiplied the biting rates by circumsporozoite rates determined by ELISA to estimate entomological inoculation rates (EIRs) for each of $P$. falciparum, $P$. vivax and $P$. malariae and then used these estimates to investigate how short-term variation in EIR affected the prevalence of infection in different age-groups. Finally, we compared these effects with those of UTN coverage.

\section{Methods}

Study area and malariological surveys

The present study comprises supplementary analyses of malariological survey data collected during 1990-92 as part of the Malaria Vaccine Evaluation and Epidemiology Project in the Wosera area of Papua New Guinea. Malaria parasitaemia is perennial with an overall prevalence of $60 \% ; 55 \%$ of infections are due to $P$. falciparum, $25 \%$ to $P$. vivax and $20 \%$ to $P$. malariae (for details see GENTON et al., 1995). Six different malaria vectors are present (HII et al., 1997).

Antimalarials are available mainly from 3 health centres and 6 aid posts, but both chloroquine and amodiaquine resistance are common (our unpublished data; SAPAK et al., 1991).

House positions were recorded using a hand-held global positioning system device (Trimble Navigational Europe, London) and validated against sketch maps. Bednet coverage (C) was quantified by the proportion of questionnaire responses, from houses within a radius of $25 \mathrm{~m}$, that indicated UTN use the previous night [see $\mathrm{HII}$ et al. (2001)].

Between July 1990 and July 1992 a total of 10001 blood slides were prepared from 7 , approximately 4monthly, sample surveys of 10 villages (GENTON et al., 
1995). During these surveys, randomly chosen individuals were identified according to previously obtained demographic information, and interviewed on UTN usage for the previous night, history of illness and drug consumption during the previous week.

\section{Estimation of entomological inoculation rate (EIR)}

The EIR is the average number of human-biting anophelines collected per person per night $(m a)$ multiplied by $s$, the proportion with sporozoites.

To obtain estimates of the EIR, all houses with recorded positions within $80 \mathrm{~m}$ of each other were grouped into a single cluster, which was assigned the locution of the centroid of the positions of the component houses. Each cluster was assigned a distinct estimate of ma derived from conditional autoregressive (CAR) models, fitted to the indoor landing rate data as described previously (SMITH et al., 1995; HII et al., 1997) by smoothing cluster data over a radius of $500 \mathrm{~m}$. Separate models were fitted for each Anopheles species and for each inter-survey interval (approximately 4 months). A lag period of 2 weeks was applied to allow for pre-patent periods. The mosquito densities associated with any one parasitological observation were thus the average expected density over the interval starting 2 weeks before the previous survey and ending 2 weeks before the current survey, and allowing for heterogeneities in the sampling effort

Sporozoite positivity was determined separately for each anopheline species by an ELISA test for circumsporozoite antigen in the head and thorax (for details see HII et al., 2001). Estimates of $s$ for $P$. falciparum and $P$. vivax were derived from logistic regression models fitted to the observed proportion of infective mosquitoes as a function of C (HII et al., 2001), separately for each vector species. For $P$. malariae, for which there was no significant relationship between UTN coverage and sporozoite rates, a single value [taken from the results of HII et al. (2001)] was assumed for the sporozoite rate for each anopheline species.

\section{Statistical models for age-prevalence curves}

The empirical age-prevalence curves were rather irregular when broken down according to categories of UTN coverage, making it difficult to compare categories and leading to uncertainties in the peak prevalences and the ages at which these prevalences were achieved. A simplified version of the model of ARON (1983) was fitted to smooth the estimated age-prevalence curves. This family of models was chosen empirically because it gave much better fits to the age-prevalence curves than did simple spline-smoothing techniques, although it is known that the model of anti-malaria immunity which these models estimate is not realistic (ANDERSON \& MAY, 1992).

We ignored host mortality (which is small compared with parasite tumover rates), but treated the host population as consisting of susceptible, infected and immune individuals in the proportions $x: y: z$, respectively where:

$$
\begin{aligned}
& \mathrm{d} x / \mathrm{d} a=\gamma z-\lambda x \\
& \mathrm{~d} y / \mathrm{d} a=\lambda x-\gamma y \\
& \mathrm{~d} z / \mathrm{d} a=\gamma y-v z
\end{aligned}
$$

Since we justify the use of the model because of its fit to the data, rather than because the parameter estimates were meaningful, we remark here only that $\lambda$ was originally intended to be the force of infection, and $\gamma$ and $v$ to quantify anti-parasite immunity. To fit this model, the boundary condition $x=1 ; y=0 ; z=0$ was assumed for age $a=0.5$ years (to allow for maternal immunity) and the expected values of $x, y, z$ at each subsequent age, $a$, conditional on the vector of parameter estimates, were computed by numerical integration using the Adams-Gear algorithm (GEAR, 1977). The maximum likelihood solutions for the parameters $\lambda, v$ and $\gamma$ were computed from the expected value of the agespecific prevalence, $y /(x+y+z)$, assuming binomial errors. Possible effects of UTN coverage on the ageprevalence curves were incorporated by allowing parameters $\lambda, \gamma$ and $v$ to vary with $C$. The parameters were taken to be linear functions of $C$, since preliminary analyses did not show any significant deviations from linearity. The statistical significance of effects of UTN coverage was tested using likelihood ratio (I,R) tests and the most appropriate model was determined via a backward elimination procedure.

Conventional logistic regression analyses were also used to test the statistical significance of UTN coverage, logarithmically transformed EIR, and age as determinants of parasite prevalence. Models including random effects terms to allow for correlation between successive observations on the same individual indicated that such within-host correlations do not have any substantial effects on these analyses.

\section{Results}

A total of 6249 blood-slide readings were analysed, corresponding to 2355 different individuals whose house positions were geocoded and hence could be included in the analysis. $P$. falciparum was found in $30.0 \%, P$. vivax in $18.8 \%$ and $P$. malariae in $13.9 \%$ of these slides.

The average EIR estimate for each malaria species is shown in Table 1 . Although UTN coverage is highest in the centre of the study area (HII et al., 2001), neither EIR estimates for $P$. falciparum (Fig. 1) not those for the other species (not shown) showed any obvious spatial pattern. Prevalence of all 3 Plasmodium species tended to be highest in the south-west of the study area (Fig. 2).

There was surprisingly little relationship between prevalence and the estimated EIR. The logistic regression analyses indicated that the EIR estimate for $P$. falciparum was related to prevalence of this species, but prevalence increased by only a factor of 1.3 for every 10 fold increase in EIR. $P$. malariae showed a similar relationship (Table 1). Analysis of interactions indicated that these effects were not significantly dependent on either age or the UTN coverage (Table 1). There was no significant relationship between the recent EIR and prevalence of $P$. vivax.

Prevalence of each malaria species varied by age, and in $P$. falciparim also depended on the coverage of bednets within a $25-\mathrm{m}$ radius (Fig. 3). The effect of UTN coverage was substantially greater than that of EIR (Table 1 and Fig. 3). The effects of increasing UTN coverage on $P$. falciparum prevalence were highest in the younger age-groups, with only small effects in adults. There was a clear tendency for peak prevalence to decrease, and occur at a greater age, as UTN coverage increased. For $P$. vivax, UTN coverage had overall no statistically significant effect on prevalence, although analysis of the age-coverage interaction suggested that coverage was associated with reduced prevalence in very young children, but possibly even an increase in prevalence in the old. For $P$. malariae, UTN coverage was assuciated with a substantial reduction in prevalence, but this reduction was not significantly age-dependent.

Independent of the effects of UTN coverage and those of the EIR, the receipt of a prescription for antimalarials in the previous month was associated with substantially lower prevalence of all 3 species.

It was difficult to locate the true maxima in the ageprevalence curves (Fig. 3), since there are considerable fluctuations in them, presumably due to random variation. Attempts to smooth them with non-parametric splines gave unsatisfactory results (not shown), but curves estimated using the model of ARON (1983) (Fig. 4) gave good fits to the prevalence data. Analysis of likelihood ratios (Table 2) indicated that the most appropriate model for $P$. falciparum was one in which $\lambda$ decreased with UTN coverage, $\gamma$ increased with UTN coverage and $v$ was independent of coverage. For both 
Table 1. Logistic regression analysis of predictors of prevalence of malaria (Wosera, Papua New Guinea, 1990-92)

\begin{tabular}{|c|c|c|c|c|}
\hline & & P. falciparum & P. vivax & P. malariae \\
\hline $\begin{array}{l}\text { EIR (infective } \\
\text { bites/person-year) }\end{array}$ & $\begin{array}{l}\text { Mean } \\
\text { (SD) }\end{array}$ & $35.4(34.5)$ & $12 \cdot 1(10 \cdot 9)$ & $9.6(8.9)$ \\
\hline $\begin{array}{l}\text { Increase in prevalence } \\
\text { with } 10 \text {-fold increase in } \\
\text { EIR }\end{array}$ & $\begin{array}{l}\text { Estimated } \\
\text { OR } \\
(95 \% \text { CL })\end{array}$ & $1 \cdot 28(1 \cdot 10,1 \cdot 50)$ & $1.07(0.87,1.32)$ & $1.32(1.04,1.68)$ \\
\hline $\begin{array}{l}\text { Test of relationship } \\
\text { between prevalence and } \\
\log (\text { EIR) }\end{array}$ & $\begin{array}{l}\text { LR } \chi^{2} \\
\text { (P-value) } \\
1 \text { d.f. }\end{array}$ & $9 \cdot 9(0.002)$ & $0.3(0.6)$ & $5.2(0.02)$ \\
\hline $\begin{array}{l}\text { Effect of antimalarial } \\
\text { prescription in previous } \\
\text { month on prevalence }\end{array}$ & $\begin{array}{l}\text { Estimated } \\
\text { OR } \\
(95 \% \mathrm{CL})\end{array}$ & $0.55(0.41,0.75)$ & $0 \cdot 28(0.17,0.44)$ & $0.13(0.05,0.36)$ \\
\hline $\begin{array}{l}\text { Test of relationship } \\
\text { between prevalence and } \\
\text { antimalarial prescription }\end{array}$ & $\begin{array}{l}\mathrm{LR} \chi^{2} \\
(P \text {-valuc }) \\
1 \mathrm{~d} . \mathrm{f} .\end{array}$ & $16.0(<0.001)$ & $39.9(<0.001)$ & $31.6(<0.001)$ \\
\hline $\begin{array}{l}\text { Test of relationship } \\
\text { between prevalence and } \\
\text { C }\end{array}$ & $\begin{array}{l}\text { LR } x^{2} \\
\text { (P-value) } \\
1 \text { d.f. }\end{array}$ & $90.9(<0.001)$ & $0.1(0.7)$ & $34.2(<0.001)$ \\
\hline $\begin{array}{l}\text { Test of age dependence } \\
\text { of relationship of } \mathrm{C} \text { to } \\
\text { prevalence }\end{array}$ & $\begin{array}{l}\text { LR } \chi^{2} \\
(P \text {-value }) \\
5 \text { d.f. }\end{array}$ & $53.1(<0.001)$ & $14 \cdot 7(0 \cdot 01)$ & $5 \cdot 7(0 \cdot 3)$ \\
\hline
\end{tabular}

EIR, entomological inoculation rate; $\mathrm{C}$, bednet coverage; $\mathrm{OR}$, odds rario; $\mathrm{CL}$, confidence limits; $\mathrm{d}$. ., degrees of freedom; $\mathrm{LR}$, likelihood ratio.

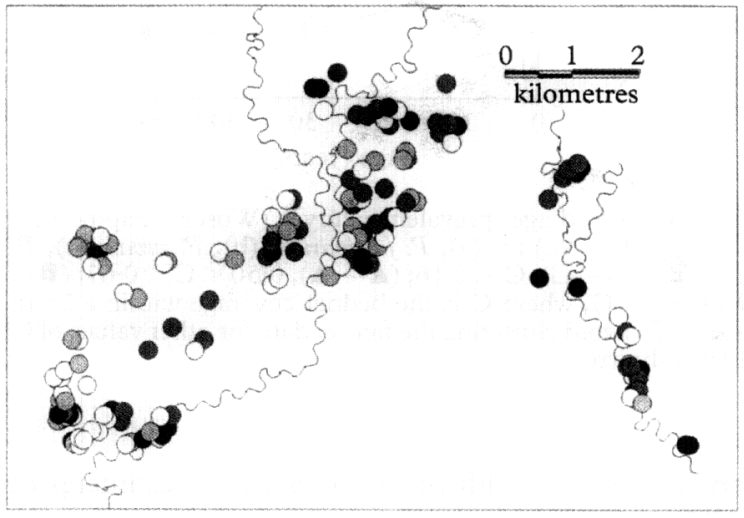

Fig. 1. Estimated average annual EIR for $P$. falciparm by cluster in the Wosera area of Papua New Guinea (1990-92). Annual number of infective bites/person: $(\Theta),>40$; (O), 20 40 ; $5-20 ;(0), 0-5$.

$P$. vivax and $P$. malariae the final model was also one in which $\lambda$ decreased with coverage, but for these species neither $\gamma$ nor $\gamma$ was dependent on $C$. Table 3 gives parameter estimates for these models.

For all 3 malaria species, the peak prevalence determined from the best-fitting age-prevalence curves decreased as UTN coverage increased (Fig. 5a), whilst the age of peak prevalence increased (Fig. 5 b). In the case of $P$. falciparum, the age of peak prevalence increased from 4.7 years $(C=0 \%)$ to $11.6(C=100 \%)$, whereas there were smaller changes for $P$. vivax (from 3.4 to 4.9 years) and for $P$. malariae ( 11.0 to 16.8 years). For $P$. falciparum the peak prevalence at $100 \%$ coverage was about half of that at zero coverage. Proportionately, the effect of UTN coverage on peak prevalence of $P$. malariae was less. The age of peak prevalence for $P$. vivax was the lowest and this species was also anomalous in that there was minimal effect of coverage on age of peak prevalence. Figure $5 \mathrm{c}$ summarizes these observations as the estimated relationship between peak prevalence and the age of peak prevalence for each species. The strength of the peak shift is estimated by considering first the length and then the gradient of each curve. Thus, $P$. falciparum showed the greatest shift, followed by $P$. malariae and finally $P$. vivax.

\section{Discussion}

As in the nearby Madang province (CATTANI et al., 1986; BURKor et al, 1987), spatial distributions of the major malaria vectors in the Wosera area of Papua New Guinea are highly focal (HIr et al., 1997), and there is extreme spatial variation in malaria transmission and prevalence. Unlike in Madang, UTN coverage (C) not vector density is the main determinant of $P$. falciparum and $P$. malarae prevalence. Neither $\mathrm{C}$, nor entomologically defined exposure, explained the distribution of $P$. vizax in the Wosera population.

Characteristically, in high-endemicity areas, variations in exposure affect mainly children (MOLINEAUX \& GRAMICCIA, 1980). In keeping with this, C measures average exposure over long periods, and its effect on prevalence of $P$. falcipanum was strikingly age-dependent (Figs 3 and 4 ) with the biggest differences in children aged 2-9 years. In contrast, prevalences of both $P$. falciparum and $P$, malariae infection were related to the estimates of EIR (which measures recent exposure) in a largely age-independent way. This finding is what we would expect if acquired immunity has little effect on infection rates, so that individual inoculations transiently add to point prevalence irrespective of the age or immunological status of the host. Slow accumulation of small effects explains why the maximum effect on prevalence is in older children. The resulting contrast in age-prevalence patterns for $P$. falcipamim between areas of low and high UTN coverage is similar to that between holo-endemic and meso-endemic villages in Senegal (RogIER \& TRAPE, 1995),

Slow accumulation of effects on prevalence could also explain the absence of efficacy against infection of UTNs in the RCT of SNOW et al. (1988). Most RCTs of ITNs in areas of high endemicity have reported only modest effects on prevalence (reviewed by LENGELER, 1998), even where transmission and disease incidence were reduced substantially (DYE et al,, 1996). Patterns of net distribution during a trial are necessarily different 

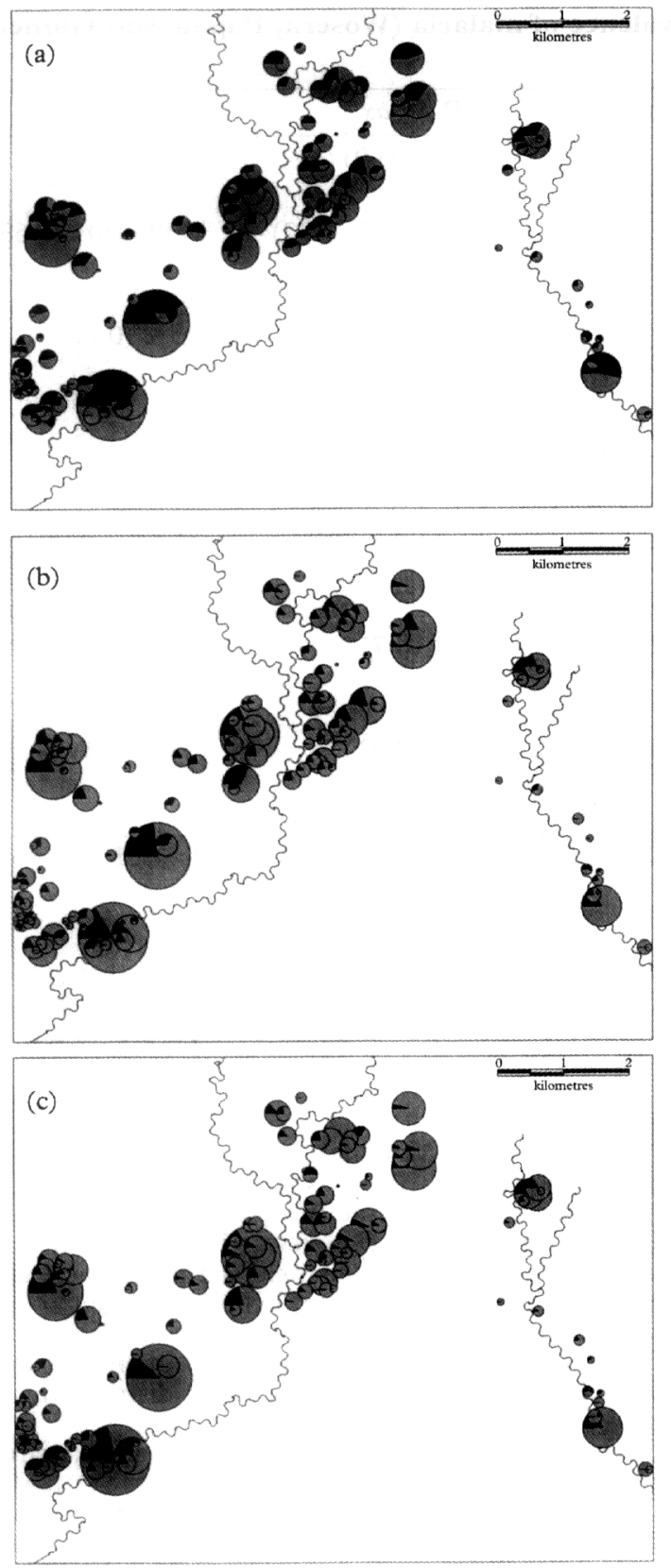

Fig. 2. Prevalence of parasitaemia by cluster (Wosera, Papua New Guinea; 1990-92) for (a), P. falciparum; (b), $P$. vivax; (c), $P$, malariae, (U), parasite positive; ( D), parasite negative. The area of the pies is proportional to the population registered in each cluster at the end of 1991 . Thin lines correspond to rivers [see HII et al. (1997) for names of villages and rivers].

from those beforehand, and randomization cannot be maintained long enough to allow estimation of effects of long-term use.

Smoothing of age-prevalence curves made it possible to visualize the shifts in age and value of peak infection prevalence associated with U'TN coverage. While overall prevalence appeared as only a poor indicator of intensity of transmission (because prevalence in adults was little affected by C), age of peak prevalence and the estimated prevalence at that age were very sensitive indicators. Age and value of peak prevalence are used as measures of endemicity of several other parasites (WoOLHOUSE, 1998), but have been little used in malariology. This may be because malaria prevalence data are generally aggregated into fairly wide age-groups and stochastic
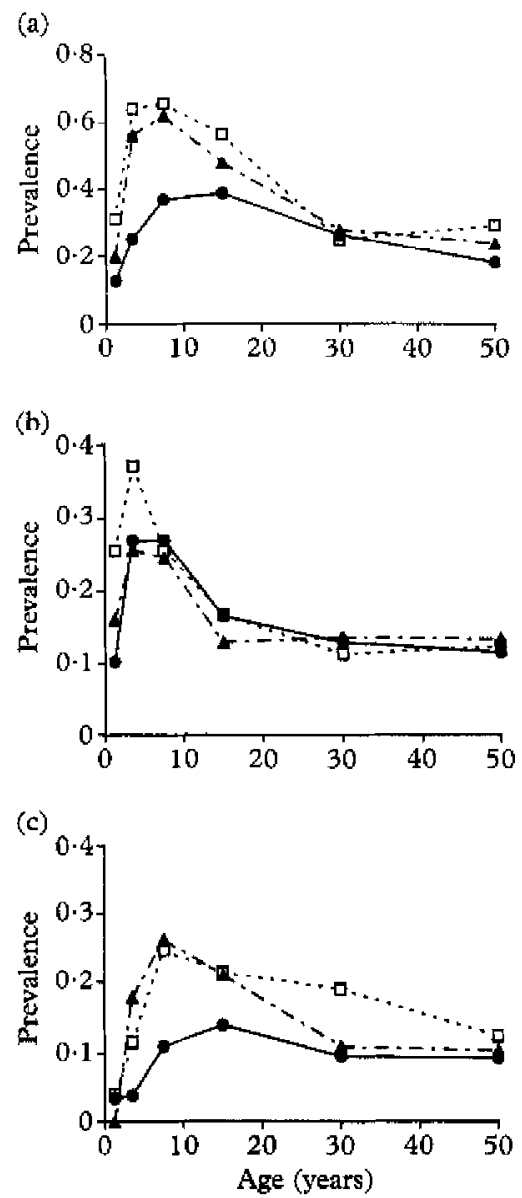

Fig. 3. Observed age-prevalence curves (Wosera, Papua New Guinea; 1990-92) for (a), $P$, falciparum; (b), $P$. vivax; (c), $P$. malariae. $(\square---\square), C<0.16 ;(\Delta-. . \Delta), 0.50<C<0.67$; b), $0.84<\mathrm{C}$, where $\mathrm{C}$ is the bednet coverage within a $25-\mathrm{m}$ radius. To avoid cluttering the figure, data for other values of $\mathrm{C}$ are not shown.

variation makes it difficult to obtain good estimates of peaks.

The extents of the peak shifts indicate the nature of acquired immunity to the 3 malaria species. Generally, when age-related data are stratified by an exposure variable, a peak shift is evidence for exposure-dependent acquisition of immunity. In the present analysis the strongest peak shift was found for $P$. falciparum, suggesting that exposure is the most important determinant of the rate at which immunity is acquired to this species.

The early maximum in the age-prevalence curve for $P$. vivax together with the very limited peak shift suggests that immunity to this species is acquired very rapidly, largely independently of transmission intensity. The inoculation rate for $P$. vivax is high enough to ensure that most children are infected very early in life and it secms possible that immunity against parasites of this species is rapidly acquired and subsequently continually boosted by relapses. Standard treatment guidelines in Papua New Guinea do not recommend radical cure of $P$. vivax and, since the relapses arise from hypnozoites, their occurrence is little dependent on the local inoculation rate.

While the EIR for $P$. malariae was similar to that of $P$. vivax, the age of peak prevalence was higher than for either of the other 2 species. This was combined with generally low infection prevalences, and a low degree of convexity in the age-prevalence profiles. Overall, these features led to an intermediate degree of peak shift, implying that exposure does play a role in the acquisition 


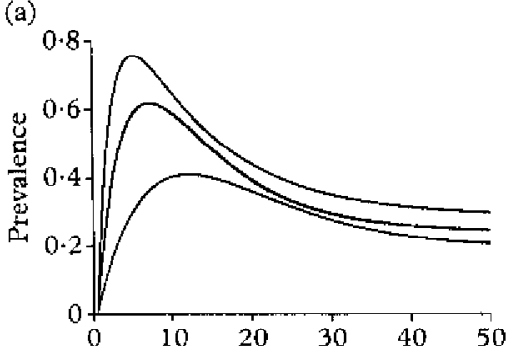

(c)

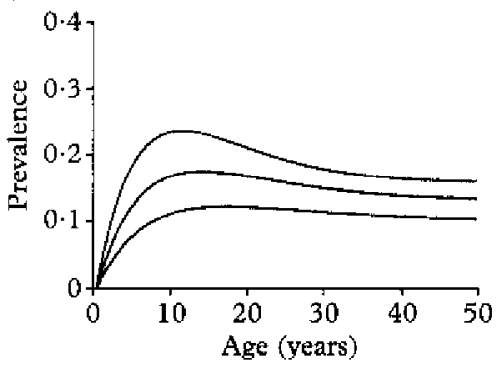

(b)

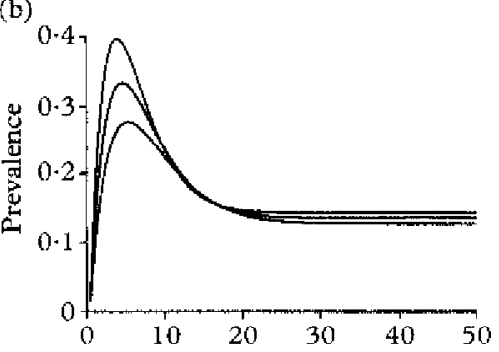

Fig. 4. Fitted age-prevalence curves (Wosera, Papua New Guinea; 1990-92) for (a), P. falciparum; (b), P. vivax; (c), $P$. malariae. I.ines correspond to $\mathrm{C}$ (bednet coverage within a 25 -th radius) $=0.0$ (highest prevalence), 0.6 and 1.0 (lowest).

Table 2. Likelihood ratio tests comparing the final model for age-prevalence curves with the full and minimal models

\begin{tabular}{|c|c|c|c|c|c|c|c|}
\hline \multirow[b]{2}{*}{ Species } & \multirow[b]{2}{*}{ Best-fitting model } & \multicolumn{3}{|c|}{ Minimal model (all constant) } & \multicolumn{3}{|c|}{ Full model (all linear) } \\
\hline & & $\underset{\text { statistic }}{\mathrm{LR}}$ & d.f. & $P$-value & $\begin{array}{l}\mathrm{LR} \\
\text { statistic }\end{array}$ & d.f. & $P$-value \\
\hline P. falciparum & $\begin{array}{l}\lambda \text { and } \gamma \text { linear with } C \\
\nu \text { constant }\end{array}$ & $183 \cdot 3$ & 2 & $<0.001$ & 0.02 & 1 & 0.9 \\
\hline$P$. vivax & $\lambda$ linear with $C$ & $12 \cdot 5$ & 1 & $<0.001$ & $1 \cdot 4$ & 2 & $0 \cdot 2$ \\
\hline P. malariae & $\begin{array}{l}\lambda \text { lincar with } C \\
\gamma, v \text { constant }\end{array}$ & $49 \cdot 6$ & 1 & $<0.001$ & 1.9 & 2 & $0 \cdot 2$ \\
\hline
\end{tabular}

See the text for explanations of the methods used.

Table 3. Parameter estimates from the final models for age-prevalence curves

\begin{tabular}{llll}
\hline Parameter & \multicolumn{1}{c}{ P. falciparum } & \multicolumn{1}{c}{ P. oivax } & \multicolumn{1}{c}{ P. malariae } \\
\hline$\lambda$ & $0.529-0.430 \mathrm{C}$ & $0.316-0.158 \mathrm{C}$ & $0.063-0.038 \mathrm{C}$ \\
$\gamma$ & $0.060-0.022 \mathrm{C}$ & 0.271 & 0.143 \\
$\nu$ & 0.026 & 0.054 & 0.048 \\
\hline
\end{tabular}

Units are per year. See the text for details of the methods used.

of immunity to this species, but that the level of exposure was insufficient to confer substantial immunity.

Our results confirmed that an adequate fit of the modified ARON (1983) model does not necessarily mean that it is an appropriate description of the underlying processes. By analysing the same dataset longitudinally, GENTON et al. (1995) estimated that the force of infection for $P$. falciparum is of the order of 2 per year. This compares with an estimate of $\lambda$ between 0.10 ( $100 \%$ UTN coverage) and 0.53 (zero UTN coverage) derived from the present model. The higher rates observed by GENTON et al. (1995) also accord with those estimated from longitudinal data in other highly endemic areas (BEKESSY et al., 1976; SMITH et al., 1999a). Transition rates estimated from studies of tumover of individual infections defined by polymerase chain reaction (PCR) are even higher (SMITH et al., 1999b).

The limited sensitivity of malaria microscopy raises a further problem with the interpretation of the simplified ARON (1983) models. Namely, that microscopy considerably underestimates the prevalence. PCR gives con- siderably higher $P$. falciparum prevalence estimates in Wosera than does microscopy, especially in older individuals (FELGER et al., 1995). In $P$. vivax the existence of a hypnozoite stage further complicates the dynamics of peripheral parasitaemia, leading to long-term cryptic infection with recurring relapses. The model makes no attempt to capture this process. The dynamics of $P$. malariae infection are not well understood, but this species is also known to be very frequently cryptic.

In a companion paper, analysing vector bionomics, we consider whether peculiarities of the local vector biology explain the impact of the UTNs (HII et al., 2001). As regards practical consequences, our results indicate that even a moderate coverage of UTNs can delay the achievement of peak prevalence and decrease the peak value for both $P$. falciparum and $P$. malariae, even when nets are not insecticide-impregnated. Even incomplete coverage of non-impregnated nets could thus have important public health implications. However, these implications, in terms of rates of malaria morbidity and mortality in Wosera, remain to be established. 
(a)

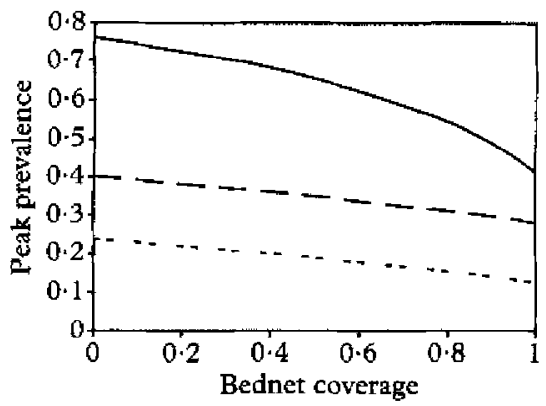

(c)

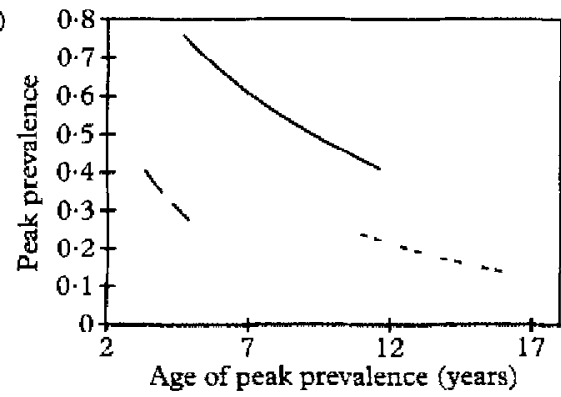

\section{Acknowledgements}

We thank the Wosera villagers for generously participating and the staff of Kunjingini Health Centre and the Papua New Guinea Institute of Medical Research in Madang and Maprik for technical support. Neal Alexander and Allan Schapira provided useful comments on an earlier draft. The Medical Research Advisory Committee of Papua New Guinea gave clearance. United States Agency for International Development grant no. 9365967.89 supported field and laboratory work. Swiss National Science Foundation Grants 32-43427.95 and 31-52984.97 supported data analysis and interpretation.

\section{References}

Anderson, R. M. \& May, R. M. (1992). Infectious Diseases of Humans. Dynamics and Control. Oxford: Oxford University Press.

Aron, J. L. (1983). The dynamics of immunity boosted by exposure to infection. Mathematical Biosciences, 64, 249-259.

Bekessy, A., Molineaux, L. \& Storey, J. (1976). Estimation of incidence and recovery rates of Plasmodtum falciparum parasitaemia from longitudinal data. Bullenth of the World Health Organization, 54, 685-691.

Burkot, T. R., Graves, P. M., Cattani, J. A., Wirtz, R. A. \& Gibson, F. D. (1987). The efficiency of sporozoite transmission in the human malarias, Plasmodim falctparum and $P$. vivax. Bulletin of the World Health Organization, 65, 375-380.

Campbell, H., Byass, P. \& Greenwood, B. M. (1987). Bed-nets and malaria suppression. Lancet, ii, 859-860.

Cattani, J. A., Moir, J. S., Gibson, F, D., Ginny, M., Paino, J., Davidson, W. \& Alpers, M. P. (1986). Small-area variations in the epidemiology of malaria in Madang Province. Papua New Guinea Medical fournal, 29, 11-17.

Dye, C., Lines, J. D. \& Curtis, C. F. (1996). A test of the malaria strain theory. Parasitology Today, 12, 89-90

Felger, I., Tavul, L., Narara, A., Genton, B., Alpers, M. \& Beck, H. P. (1995). The use of the polymerase chain reaction for more sensicive detection of Plasmodium falciparum. Papua New Guinea Medical foumal, 38, 52-56.

Gear, C. W. (1977). Numerical Initial Value Problems in Ordinary Differential Equations. Englewood Cliffs, New Jersey: Prentice Hall.

Genton, B., Hii, J., Al-Yaman, F., Paru, R, Beck, H.-P., Ginny M., Dagoro, H., Lewis, D. \& Alpers, M. P. (1994). The use of untreated bednets and malaria infection, morbidity and immunity, Annals of Tropical Medicine and Parasitology, 8 $263-270$.

Genton, B., Al-Yaman, F., Beck, H.-P., Hii, J., Mellor, S. Narara, A., Gibsun, N., Smith, T. \& Alpers, M. P. (1995) The epidemiology of malaria in the Wosera area, East Sepik Province of Papua New Guinea, in preparation for vaccine trials. I. Malariometric indices and immunity. Annals of Tropical Medicine and Parasitology, 89, 359-376.

Hii, J., Smith, T., Mai, A., Mellor, S., Lewis, D., Alexander, N.

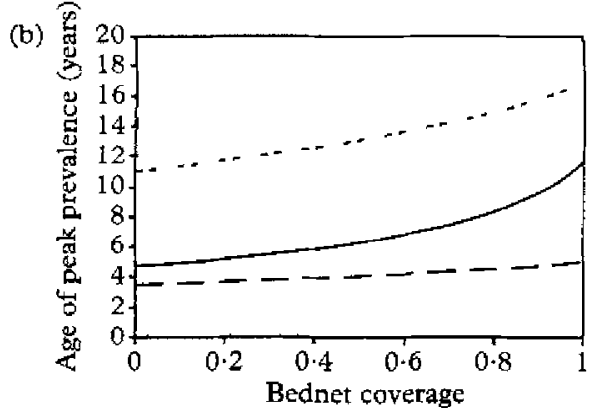

Fig. 5. Relarionships berween peak malaria prevalence, age of peak prevalence and bednet coverage (Wosera, Papua New Guinea; 1990-92), for different Plasmodium species. (a) Peak prevalence by bednet coverage; (b) age of peak prevalence by bednet coverage; (c) peak prevalence by age of peak prevalence. $(-), P$ falciparm; $(-\cdots), P$. vivax; $(--), P$. malariae

\& Alpers, M. P. (1997). Spatial and temporal variacion in abundance of Anopheles species (Diptera: Culicidae) in a malaria endemic area in Papua New Guinea. Foumal of Medical Entomology, 34, 212-217.

Hii, J. L. K, Smith, T, Vounatsou, P., Alexander, N., Mai, A. Ibam, E. \& Alpers, M. P. (2001). Area effects of bednet use in a malaria-endemic area in Papua New Guinea. Transactions of the Royal Society of Tropical Medicine and Hygiene, 95, 7-13.

Lengeler, C. (1998). Insecticide-treated bednets and curtains for malaria control. In: Infectious Disedses Module of The Gochrane Database of Systematic Reviews, Gatner, P., Gelband, H., Olliaro, P. \& Salizas, R. (editors). Oxford: The Cochrane Collaboration.

Lindsay, S. W. \& Gibson, M. E. (1988). Bednets revisited-old idea, new angle. Parasitology Today, 4(10), 270-272.

Molineaux, I.. \& Gramiccia, G. (1980). The Garki Project. Reseanch on the Epidemiology and Control of Malaria in the Sudan Savanna of West Africa. Geneva, Switzerland: World Health Organization.

Rogier, C. \& Trape, J.-F. (1995). Etude de l'acquisition de la prémunition en zones d'holo- et de meso-endemie palustre à Dielmo er à Ndiop (Sénégal): resultats preliminaires, 19901994. Médecine Tropicale, 55 (4 supplement), 71-76.

Sapak, P., Garner, P., Baea, M., Narara, A., Heywood, P. \& Alpers, M. (1991). Ineffectiveness of amodiaquine against Plasmodium falciparum malaria in symptomatic young children living in an endemic malarious area of Papua New Guinea. Fotmal of Tropical Paediatrics, 37, 1-6.

Smith, T., Charlwood, J. D., Takken. W., Tanner, M. \& Spiegelhalter, D. (1995). Mapping the densities of malaria vectors within a single village. Acta Tropica, 59, 1-18.

Smith, T., Beck, H.-P., Kitua, A., Mwankusye, S., Felger, I., Fraser-Hurt, N., Irion, A., Alonso, P., Teuscher, T. \& Tanner, M. (1999a). Age dependence of multiplicity of Plasmodium falciparum infection and of other malariological indices in an area of high endemicity. Transactions of the Ronral Saciery of Troprical Medicine and Hygtene, 93, supplement $1, \mathrm{Sl} /$ 15-S1/20.

Smith, T. Felger, I., Fraser-FIurt, N. \& Beck, H.-P. (1999b) Effects of insecticide-treated nets on the dynamics of multiple Plasmodium falciparum infections. Transactions of the Royal Socisy of Troptcal Medicine and Hygiene, 93, supplement $1, S 1 /$ 53-S1/57.

Snow, R. W., Rowan, K. M., Lindsay, S. W. \& Greenwood, B. M. (1988). A trial of bed nets (mosquito nets) as a malaria control strategy in a rural area of The Gambia, West Africa. Transactions of the Royal Society of Tropical Medicine and Hygiene, 82, 212-215.

Woolhouse, M. E J (1998), Patterng in parasite epidemiology: the peak shift. Parasitology Today, 14, 428-434

Received 8 September 1999; revised 16 Yune 2000; accepted for publication 20 fune 2000 